USACH-FM-00/07

\title{
Deformed Heisenberg Algebra with Reflection, Anyons and Supersymmetry of Parabosons*
}

\author{
Mikhail S. Plyushchay ${ }^{\star, \dagger}, \sharp$ \\ ${ }^{\star}$ Departamento de Física, Universidad de Santiago de Chile, \\ Casilla 307, Santiago 2, Chile \\ ${ }^{\dagger}$ Institute for High Energy Physics, Protvino, Russia \\ ${ }^{\sharp}$ E-mail: mplyushc@lauca.usach.cl
}

\begin{abstract}
Deformed Heisenberg algebra with reflection appeared in the context of Wigner's generalized quantization schemes underlying the concept of parafields and parastatistics of Green, Volkov, Greenberg and Messiah. We review the application of this algebra for the universal description of ordinary spin- $j$ and anyon fields in $2+1$ dimensions, and discuss the intimate relation between parastatistics and supersymmetry.
\end{abstract}

\section{Introduction}

Generalized statistics was introfuced in physics in the form of parastatistics as an exotic possibility extending the Bose and Fermi statistics 15 . It was closely related with the discovery of color in the context of the theory of strong interactions. Nowadays generalized statistics 9 finds applications in the physics of the quantum Hall effect and (probably) it is relevant to high temperature superconductivity 10 . Supersymmetry, instead, unifies Bose and Fermistatistics $11-15$ and its development lead to the construction of field and string theories with exceptional properties 16 , that transformed the same idea of supersymmetry in one of the cornerstones of modern theoretical physics. Supersymmetry was observed in nuclear physics in the form of dynamical symmetry 17,18 , whereas its manifestation as a fundamental symmetry of elementary particle physics still waits for experimentaleonfirmation.

Though supersymmetry and generalized statistics may be unified in the form of parasupersymmetry 19 , nevertheless, by the construction, the two concepts seem to be independent. Recently, the existence of intimate relation between generalized statistics and supersymmetry was established by observation of hidden supersymmetric structure in purely parabosonic20 and purely pafermionid21 systems. The key tool with which the observation of close relationship between generalized statistics and supersymmetry was realized is the so called deformed Heisenberg algebra with reflection, or the R-deformed Heisenberg algebra (RDHA)22, 23. The algebraic construction of RDHA appeared in the work of Wigner24, where he investigated the problem of correlation of equations of motion with quantum mechanical commutation relations and pfoposed the generalized quantization schemes which subsequently lead to the theoretical discovery of parastatisticst 5 (in this context, see also refs.25 28). RDHA represents, probably, one of the first examples of deformation of bosonicharmonic oscillator which, as it sas sown recently, possesses some universality being also related to parafermipns22.23, to $(2+1)$-dimensional anyons 2931 , and to the bosonized form of supersymmetricquantum mechanics 32 30.33. Besides, the RDHA structure underlies the construction of fractional supersymmetry 34 . In this talk we review the application of RDHA for the universal description of ordinary spin- $j$ and anyon fields in $2+1$ dimensions by means of first order linear differential equations 29 , and discuss the exotic supersymmetry of purely parabosonic systems 20 .

\footnotetext{
${ }^{*}$ Talk given at the International Conference on "Spin-Statistics Connection and Commutation Relations", Centro Internazionale per la Cultura Scientifica dell'Università di Napoli, Anacapri, Capri Island, Italy - May 31-June 3, 2000
} 


\section{Supersymmetry of parabosons}

The deformed Heisenberg algebra with reflection is generated by the creation-annihilation operators $a^{+}, a^{-}$and by the reflection operator $R$ satisfying the relations

$$
\left[a^{-}, a^{+}\right]=1+\nu R, \quad\left\{R, a^{ \pm}\right\}=0, \quad R^{2}=1,
$$

where $\nu$ is a real deformation parameter. Due to these basic relations, the creation-annihilation operators satisfy the trilinear parabosonic commutation relations $\left[\left\{a^{-}, a^{+}\right\}, a^{ \pm}\right]= \pm 2 a^{ \pm}$. Introducing the Fock vacuum state, $a^{-}|0\rangle=0$ and fixing the action of operator $R$ on it as $R|0\rangle=|0\rangle$, we arrive at the relation $a^{-} a^{+}|0\rangle=(1+\nu)|0\rangle$, which together with trilinear commutation relation means that at $\nu=p-1, p=1,2, \ldots$, the operators $a^{ \pm}$have the sense of single-mode creation-annihilation operators of paraboson of order $p$. Vice versa, one can show that parabosonic trilinear commutation relations themselves give rise to RDHAE2. In general case, the number operator is realized in the form $N=\frac{1}{2}\left\{a^{-}, a^{+}\right\}-\frac{1}{2}(1+\nu)$, and the reflection operator can be represented as $R=(-1)^{N}=\cos \pi N$.

The reflection operator introduces a natural $Z_{2}$ grading structure in the Fock space and its presence in the definition of RDHA can be considered as an indication on possible relationship between parabosons and supersymmetry. To reveal a supersymmetry of parabosons, one notes that RDHA can also be given by the relations 20

$$
a^{+} a^{-}=F(N), \quad a^{-} a^{+}=F(N+1), \quad\left[N, a^{ \pm},\right]= \pm a^{ \pm},
$$

where $F(N)=N+\nu \sin ^{2} \frac{\pi N}{2}$ is the characteristic function satisfying for $\nu>-1$ the relations $F(0)=0, F(n)>0$, $n=1,2, \ldots$. These relations mean, in particular, that for $\nu>-1$ the corresponding representations of RDHA are unitary and infinite-dimensional. On the other hand, in the case $\nu=-(2 p+1), p=1,2, \ldots$, the characteristic function possesses the property $F(2 p+1)=0$ underlying the existence of $(2 p+1)$-dimensional (non-unitary) irreducible representations of RDHA, which are associated with the deformed parafermions of order $2 p=2,4, \ldots$.22. 23. 21. Let us restrict ourselves here by the case of unitary infinite-dimensional representations $(\nu>-1)$. When $\nu=2 k+1$, $k=0,1, \ldots$, the structure function satisfies the relation $F(2 n+1)=F(2 n+\nu+1), n=0,1, \ldots$ Therefore, in the case of parabosonic systems of even order $p=2(k+1)$, the spectrum of the quadratic Hamiltonian $H=a^{+} a^{-}$(or of $H=a^{-} a^{+}$), reveals doubling of all higher-lying levels. This indicates on existence of supersymmetry in such purely parabosonic systems. As it follows from the explicit form of the characteristic function, in the case of paraboson of order $p=2(k+1), k=0,1,2 \ldots$, and Hamiltonian $H=a^{+} a^{-}$, the supersymmetry is characterized by the presence of $k+1$ singlet states with energies $E=0,2, \ldots, 2 k$. Therefore, only in the case $k=0$, the spectrum has one singlet state of zero energy, whereas all other cases are characterized by the presence of $k$ higher-lying singlet states of nonzero energy in addition to the zero energy ground state. The corresponding supercharges are the infinite series operators in the corresponding paraboson operators:

$$
Q_{+}=\left(a^{+}\right)^{2 k+1} \sin ^{2} \pi J_{0}, \quad Q_{-}=\left(a^{-}\right)^{2 k+1} \cos ^{2} \pi J_{0}, \quad J_{0}=\frac{1}{4}\left\{a^{+}, a^{-}\right\} .
$$

They together with the Hamiltonian satisfy the polynomial superalgebra:

$$
\begin{gathered}
Q_{ \pm}^{2}=0, \quad\left[H, Q_{ \pm}\right]=0, \\
\left\{Q_{+}, Q_{-}\right\}=(H-2 k)(H-2 k+2) \ldots(H+2 k-2)(H+2 k),
\end{gathered}
$$

which in the case $k=0$ is reduced to the conventional $N=1$ linear superalgebra. The role of the grading operator in such purely parabosonic supersymmetric systems belongs to the reflection operator $R=\cos \pi N$.

The case of paraboson system of order $p=2(\nu=1)$ given by the Hamiltonian $H=a^{-} a^{+}$is characterized by the $N=1$ spontaneously broken linear supersymmetry: all the states are paired in supersymmetric doublets with the lowest energy level $E=2$. The corresponding supercharges have the form (3) with $k=0$ and with operators $a^{+}$and $a^{-}$changed in their places 32 . The systems of parabosons of order $p=4,6, \ldots$ given by the Hamiltonian $H=a^{-} a^{+}-2$ possessnonlinear (polynomial) supersymmetry of the form similar to (4) 20 .

It was shown in ref.20 that the supersymmetry of purely parabosonic systems can be understood as the supersymmetry of Calogero-like systems with exchange interaction and that in principle it can be realized in one-dimensional systems of identical fermions. Besides, it was demonstrated that nonlinear parabosonic supersymmetry can be obtained via appropriate modification of the classical analog of usual supersymmetric quantum mechanics. 


\section{RDHA and anyons}

The parabosonic supersymmetry structures corresponding to $H=a^{+} a^{-}$and $H=a^{-} a^{+}$can be unified and extended to the $\operatorname{osp}(2 \mid 2)$ superalgebraic structure 32 . The operators $T_{3}=\frac{1}{2}\left\{a^{+}, a^{-}\right\}, T_{ \pm}=\frac{1}{2}\left(a^{ \pm}\right)^{2}$ and $I=\frac{1}{2}(\nu+R)$ have a sense of even generators of $\operatorname{sep}(2 \mid 2)$ forming $\operatorname{sl}(2) \times u(1)$ subalgebra, whereas the operators $Q^{ \pm}=a^{ \pm} \Pi_{ \pm}$and $S^{ \pm}=a^{ \pm} \Pi_{\mp}$ are its odd generators, where $\Pi_{ \pm}=\frac{1}{2}(1 \pm R)$ are the projectors on even and odd subspaces of the Fock space. On the other hand, the operators $J_{\mu}, \mu=0,1,2, J_{0}=\frac{1}{2} T_{3}, J_{1} \pm i J_{2}=T_{ \pm}$, and $\mathcal{L}_{\alpha}, \alpha=1,2$, $\mathcal{L}_{1}=\left(a^{+}+a^{-}\right) / \sqrt{2}, \mathcal{L}_{2}=i\left(a^{+}-a^{-}\right) / \sqrt{2}$, can be considered as even and odd generators of $\operatorname{osp}(1 \mid 2)$ superalgebra: $\left[J_{\mu}, J_{\nu}\right]=-i \epsilon_{\mu \nu \lambda} J^{\lambda},\left[J_{\mu}, \mathcal{L}_{\alpha}\right]=\frac{1}{2}\left(\gamma_{\mu}\right)_{\alpha}{ }^{\beta} \mathcal{L}_{\beta},\left\{\mathcal{L}_{\alpha}, \mathcal{L}_{\beta}\right\}=4 i(J \gamma)_{\alpha \beta}$; here $(2+1)$-dimensional $\gamma$-matrices appear in the Majorana representation, see ref.29. Since the Casimir operator $\mathcal{C}=J_{\mu} J^{\mu}-\frac{i}{8} \mathcal{L}^{\alpha} \mathcal{L}_{\alpha}$ takes the fixed value $\mathcal{C}=\frac{1}{16}\left(1-\nu^{2}\right)$, this means that any irreducible representation of RDHA carries the corresponding irreducible representation of $\operatorname{osp}(1 \mid 2)$, which, in turn, is a direct sum of two irreducible representations of $s o(2,1)$ with the Casimir operator $C=J^{\mu} J_{\mu}$ taking the value $C=-\alpha_{+}\left(\alpha_{+}-1\right), \alpha_{+}=\frac{1}{4}(1+\nu)$, on even $(R=1)$ subspace of the Fock space, and $C=-\alpha_{-}\left(\alpha_{-}-1\right), \alpha_{-}=\alpha_{+}+\frac{1}{2}$, on odd $(R=-1)$ subspace. The osp $(1 \mid 2)$ structure associated with RDHA can be exploited to describe anyons by means of covariant linear differential equations. For the purpose, let us consider the field $\Psi^{n}(x)$ depending on space-time point $x_{\mu}$ in $2+1$ dimensions and carrying infinite- $(\nu>-1$, $n=0,1, \ldots)$ or finite- dimensional $(\nu=-(2 p+1), p=1,2, \ldots, n=0, \ldots, 2 p)$ representation of RDHA Then the following spinor set of linear differential equations describes universally ordinary spin- $j$ fields and anyons 29 .

$$
D_{\alpha} \Psi(x)=0, \quad D_{\alpha}=R \mathcal{P}_{\alpha}+m \mathcal{L}_{\alpha}
$$

where $R$ is the reflection operator of RDHA, $m$ is a mass parameter, and $\mathcal{P}_{\alpha}=\left(-i \gamma_{\mu} \partial^{\mu}\right)_{\alpha}{ }^{\beta} \mathcal{L}_{\beta}$. The condition of integrability of two equations $(5)$ is equivalent to the equations $\left(-\partial^{2}+m^{2}\right) \Psi_{+}(x)=0,\left(-i \partial^{\mu} J_{\mu}-s m\right) \Psi_{+}(x)=0$, $s=\frac{1}{4}(1+\nu)$, for the even part (in the RDHA sense) of the field, $R \Psi_{+}(x)=\Psi_{+}(x)$, whereas the solution to equations (5) in odd subspace, $R \Psi_{-}(x)=-\Psi_{-}(x)$, is trivial, $\Psi_{-}(x)=0$. The parameter $\nu$ fixes the value of spin $s$, and one concludes that in the case of finite-dimensional representations $(\nu=-(2 p+1))$, the corresponding field $\Psi_{+}(x)$ carries integer or half-integer spin $s=j$, whereas the case of infinite-dimensional representations of RDHA $(\nu>-1)$ corresponds to the field of arbitrary spin $s>0$ (anyon). The case of anyon with $s<0$ can be obtained by a simple change $m \rightarrow-m$ in (5). In the case of infinite-dimensional unitary representations of RDHA, the linear differential equation $\left(-i \partial^{\mu} J_{\mu}-s m\right) \Psi_{+}(x)=0$ is the $(2+1)$-dimensional analog of the $(3+1) D$ infinite-component Majorana equation, whose fundamental role for the description of anyons wastestablished in ref. 35 under investigation of the $(2+1) D$ model of relativistic particle with torsion (see also refs.36.37).

Varying the deformation parameter in the region $\nu>-1$, one can obtain the fields of integer spin $(\nu=4 n-1, s=n$, $n=1,2, \ldots)$ as well as of half-integer spin $\left(\nu=4 n+1, s=n+\frac{1}{2}, n=0,1, \ldots\right)$. However, such fields of integer and half-integer spin have a nature to be essentially different from the nature of usual spin- $j$ fields appearing in the case of finite-dimensional representations of RDHA $(\nu=-(2 p+1))$ since they have hidden nonlocality. In the rest frame, the solution to the Klein-Gordon and Majorana equations has enly one nontrivial component in correspondence with the pseudoscalar (helicity) nature of spin in $2+1$ dimensions 38,39. But the Lorentz boost enlivens all the infinite number of components of the field $\Psi_{+}(x)$ in the case $\nu>-131$. There is the analog of coordinate representation for RDHA, in which

$$
a^{ \pm}=\frac{1}{\sqrt{2}}\left(q \mp i \mathcal{D}_{\nu}\right), \quad \mathcal{D}_{\nu}=-i\left(\frac{d}{d q}-\frac{\nu}{2 q} R\right),
$$

and $R \psi(q)=\psi(-q)$. In such representation the fields $\Psi_{+}(x)$ have a structure of the functions to be even in continuous variable $q$ : $\Psi_{+}(x, q)=\Psi_{+}(x,-q)$. This means that the corresponding solutions to the equations (5) in the case $\nu>-1$ have the hidden_half-infinite nonlocality $(q \geq 0)$ which is analogous to the string-like nonlocality of anyon fields in other approaches 4 .41.

To conclude, RDHA finds various theoretical applications including the described two. It would be interesting to look for the experimental manifestation of the exotic supersymmetry of parabosons. In general, the existence of the polynomial supersymmetry is characterized by the presence of several singlet states which could be considered as an indication on parabosonic-like excitations (quasiparticles) in the system. The search for possible experimental manifestation of the nonlocal (in internal variable $q$ ) fields of integer and half-integer spin associated with parabosons seems to be another interesting problem. 
Acknowledgments. I am grateful to I. Bandos, M. RauschdeTraubenberg and D. Sorokin for useful discussions, and to G. Marmo and E. C. G. Sudarshan for bringing refs.26 28 to my attention. The work was supported in part by FONDECYT (Chile) under grant 1980619 and by DICYT (USACH).

1. Green, H. S., Phys. Rev. 90, 270 (1953).

2. Volkov, D. V., Sov. JETP 9, 1107 (1959); 11, 375 (1960).

3. Greenberg, O. W., Phys. Rev. Lett. 13, 598 (1964).

4. Greenberg, O. W., and Messiah, A. M. L., Phys. Rev. B136, 248 (1964).

5. Ohnuki, Y., and Kamefuchi, S., Quantum Field Theory and Parastatistics, Tokyo: University Press, 1982.

6. Leinaas, J. M., and Myrheim, J., Nuovo Cimento 37B, 1 (1977).

7. Goldin, G. A., Menikoff, R., and Sharp, D. H., J. Math. Phys. 21, 650 (1980); 22, 1664 (1981).

8. Wilczek, F., Phys. Rev. Lett. 48, 1144 (1982); 49, 957 (1982).

9. Wilczek, F., and Zee, A., Phys. Rev. Lett. 51, 2250 (1983).

10. Wilczek, F., Fractional Statistics and Anyon Superconductivity, Singapore: World Scientific, 1990.

11. Gol'fand, Y. A., and Likhtman, E. P., JETP Lett. 13, 323 (1971).

12. Ramond, P., Phys. Rev. D3, 2415 (1971).

13. Neveu, A., and Schwarz, J., Nucl. Phys. B31, 86 (1971).

14. Volkov, D., and Akulov, V., JETP Lett. 16, 438 (1972).

15. Wess, J., and Zumino, B., Nucl. Phys. B70, 39 (1974).

16. Green, M. B., Schwarz, J., and Witten, E., Superstring Theory, Cambridge, 1987.

17. Iachello, F., Phys. Rev. Lett. 44, 772 (1980).

18. Metz, A., et al., Phys. Rev. Lett. 83, 1542 (1999).

19. Rubakov, V. A., and Spiridonov, V. P., Mod. Phys. Lett. A3, 1337 (1988).

20. Plyushchay, M., Hidden Nonlinear Supersymmetries in Pure Parabosonic Systems, Int. J. Mod. Phys. A, in press, hepth/9903130.

21. Klishevich, S., and Plyushchay, M., Mod. Phys. Lett. A14, 2739 (1999).

22. Plyushchay, M. S., Nucl. Phys. B491, 619 (1997).

23. Plyushchay, M. S., Mod. Phys. Lett. A11, 2953 (1996).

24. Wigner, E. P., Phys. Rev. 77, 711 (1950).

25. Yang, L. M., Phys. Rev. 84, 788 (1951).

26. Ryan, C., and Sudarshan, E. C. G., Nucl. Phys. B47, 207 (1963).

27. Mukunda, N., Sudarshan, E. C. G., Sharma, J. K., and Mehta, C. L., J. Math. Phys. 21, 2386 (1980); 22, 78 (1981).

28. Man'ko, V. I., Marmo, G., Sudarshan, E. C. G., and Zaccaria, F., Int. J. Mod. Phys. B11, 1281 (1997).

29. Plyushchay, M. S., Phys. Lett. B320, 91 (1994).

30. Plyushchay, M. S., Ann. Phys. 245, 339 (1996).

31. Plyushchay, M. S., Mod. Phys. Lett. A12, 1153 (1997).

32. Plyushchay, M. S., Mod. Phys. Lett. A11, 397 (1996).

33. Gamboa, J., Plyushchay, M., and Zanelli, J., Nucl. Phys. B543, 447 (1999).

34. Rausch de Traubenberg, M., and Slupinski, M. J., Mod. Phys. Lett. A12, 3051 (1997); J. Math. Phys. 41, 4556 (2000).

35. Plyushchay, M. S., Phys. Lett. B262, 71 (1991); Nucl. Phys. B362, 54 (1991).

36. Jackiw, R., and Nair, V. P., Phys. Rev. D43, 1933 (1991).

37. Plyushchay, M. S., Phys. Lett. B273, 250 (1991); Int. J. Mod. Phys. A7, 7045 (1992).

38. Cortés, J. L., and Plyushchay, M. S., Int. J. Mod. Phys. A11, 3331 (1996).

39. Plyushchay, M. S., Monopole Chern-Simons Term: Charge-Monopole System as a Particle with Spin, hep-th/0004032.

40. Buchholz, D., and Fredenhagen, K., Comm. Math. Phys. 84, 1 (1982).

41. Fröhlich, J., and Marchetti, P. A., Nucl. Phys. B356, 533 (1991). 